MPI-PhT 99-16

April

\title{
Holography based on noncommutative geometry and the AdS/CFT correspondence
}

\author{
Zhe Chang \\ Max-Planck-Institut für Physik \\ Werner-Heisenberg-Institut \\ Föhringer Ring 6, D-80805 München, Germany \\ and \\ Institute of High Energy Physics, Academia Sinica \\ P.O.Box 918(4), 100039 Beijing, China
}

\begin{abstract}
Exponential regularization of orthogonal and Anti-de Sitter (AdS) space is presented based on noncommutative geometry. We show that an adequately adopted noncommutative deformation of geometry makes the holography of higher dimensional quantum theory of gravity and lower dimensional theory possible. Detail counting for observable degrees of freedom of quantum system of gravity in the bulk of noncommutative space $S O_{q}(3)$ and the classical limit of its boundary surface $S^{3}$ is discussed. Taking noncommutivity effect into account, we get the desired form of entropy for our world, which is consistent with the physical phenomena associated with gravitational collapse. Conformally invariant symmetry is obtained for the equivalent theory of the quantum gravity living on the classical limit of boundary of the noncommutative AdS space. This is the bases of the AdS/CFT correspondence in string theory.
\end{abstract}




\section{Introduction}

By making use of the idea that a quantum system of gravity may possess far less degrees of freedom than usually expected for a $3+1$ dimensional field theory [1] [2], it was suggested recently that there is an AdS/CFT correspondence[3] - [5]: the string theory ( $\mathrm{M}$ theory) on background of the form $\mathrm{AdS}_{d} \times M_{D-d}$ is dual to a conformal field theory living on the spacetime boundary. Here $\mathrm{AdS}_{d}$ is an Anti-de Sitter (AdS) space of spacetime dimension $d$, and $M_{D-d}$ is a certain compactification space of dimension $D-d$ with $D=10$ for string theory ( $D=11$ for M theory). A strong support for the proposal comes from comparing spectra of Type IIB string theory on the background of $\mathrm{AdS}_{5} \times S^{5}$ and low-order correlation functions of the $3+1$ dimensional $\mathcal{N}=4 S U(N)$ super Yang-Mills theory. The dual super Yang-Mills theory lives on the boundary of the AdS space. To each field $\Phi_{i}$ there is a corresponding local operator $\mathcal{O}_{i}$ in the conformal field theory. The precise relation between string theory in the bulk and field theory on the boundary is

$$
Z_{\mathrm{eff}}\left(\Phi_{i}\right)=e^{i S_{\mathrm{eff}}\left(\Phi_{i}\right)}=\left\langle T e^{i \int_{\mathcal{B}} \Phi_{b, i} \mathcal{O}^{i}}\right\rangle
$$

where $S_{\text {eff }}$ is the effective action in the bulk and $\Phi_{b, i}$ is the field $\Phi_{i}$ restricted to the boundary. In the large $N$ limit, the string theory is weakly coupled and supergravity is a good approximation to it. Thus it is possible to describe a precise recipe expressing correlation functions of the $\mathcal{N}=4$ super Yang-Mills theory in four dimensions in terms of a calculations of tree approximation to supergravity performed in the bulk.

At first sight, it seems very strange for us that quantum theories in different spacetime dimensions ever could be equivalent in any sense. The key to understand it is the fact that the theory in the larger dimension is always a quantum theory of gravity. For such theories the concepts of holography has been introduced by 't Hooft [1] based on phenomenological study of the black hole theory and was referred as a generic properties of quantum system of gravity. The AdS/CFT correspondence is just an example 
of the realization of the holography on quantum theory of gravity. From point of view of general relativity, gravity is nothing but spacetime geometry. To check the Maldacena conjecture, one has to start from investigating of adequate geometry description for quantum theory of gravity. Because the holography is a generic properties of the quantum gravity, in principle, it should be deduced naturally from geometric properties of spacetime. 't Hooft showed that simple regularization of spacetime can not give correct account of observable degrees of freedom for quantum theory of gravity. On the other hand, the poor understanding of physics at Planck scale also indicates that the small scale structure of spacetime might not be adequately described by classical continuum geometry. Thus, new geometry should be introduced for quantum gravity. It has long time been suspected that the noncommutitive spacetime might be a realistic picture of how spacetime behaves near the Planck scale[6] [7]. Strong quantum fluctuations of gravity may make points in space fuzzy. The noncommutative geometry description [8][9] is a strong candidate for quantum theory of gravity. We wish the holography can be obtained explicitly based on noncommutative geometry picture of quantum gravity and show the AdS/CFT correspondence by demonstrating conformally invariant symmetry on the boundary surface of noncommutative AdS space.

In this paper, we present a kind of special regularization with exponentially increasing spacetime cutoff for both orthogonal and AdS space based on noncommutative geometry. It is used to argue that the same minimal cutoff of any geometry is the Planck scale $l_{p}$, that the most direct and obvious physical cutoff is from the formation of microscopic black holes as soon as too much energy would be accumulated into too small a region [1]. We show that an adequately adopted noncommutative deformation of geometry makes the holography of higher dimensional quantum system of gravity and lower dimensional theory possible. As an example, detail calculations are carried out for the counting of observable degrees of freedom of quantum gravity in the bulk of noncommutative space $S O_{q}(3)$ and the classical limit of its boundary surface $S^{3}$. 
Results show that a very small (may be $<10^{-15}$ ) displacement of the noncommutative deformation parameter from its classical value 1 reduces sharply the entropy of the quantum system of gravity. The desired entropy expression $S=4 \pi M^{2}+C$ of the universe can be deduced naturally. Conformally invariant symmetry is obtained for the equivalent theory of the quantum gravity, which lives on the boundary of the noncommutative AdS space. This is the bases of the AdS /CFT correspondence in nonperturbative string theory and $\mathrm{M}$ theory.

This paper is organized as follows. In Section 2, we discuss the noncommutative orthogonal space $S O_{q}(3)$. The algebra satisfied by the coordinates and derivatives is decoupled into three independent subalgebras by introducing of a new set of variables. Quantum coherent states are constructed as reference ones for investigating representations of the spacetime algebra. Forms of Hilbert space show clearly that the noncommutative spacetime is discretely latticed with exponentially increasing space distances. The noncommutative deformation parameter is determined by an algebraic equation. The noncommutative space $S O_{q}(3)$ has a same entropy or observable degrees of freedom with the classical $S^{3}$ surface. Section 3 is devoted to the study of noncommutative $\mathrm{AdS}_{2 n}$ space. Conjugate operation is set up for the noncommutative AdS space. This conjugation has a induced counterpart for the set of decoupled coordinates and derivatives. Hilbert space of the noncommutative $\mathrm{AdS}_{2 n}$ space is constructed based on quantum coherent states. Discrete lattice structure of the noncommutative $\mathrm{AdS}_{2 n}$ with exponentially increasing space distances is obtained. Holography makes the quantum system of gravity on the noncommutative $\mathrm{AdS}_{2 n}$ space equivalent to the conformally invariant quantum theory living on the classical limit of its boundary surface. This is crucial for the AdS/CFT correspondence of string theory and M theory. By almost same procedures, properties of the noncommutative $\operatorname{Ad}_{2 n-1}$ space are shown in Section 4. In Section 5, some concluding remarks are given. 


\section{Noncommutative geometry and holography}

We begin by discussing the quantum space $S O_{q}(3)$ with coordinates $x^{i}(i=-, 0,+)$. The commutation relations 10 among coordinates $x^{i}$ are

$$
\begin{aligned}
& x^{-} x^{0}=q x^{0} x^{-}, \quad x^{0} x^{+}=q x^{+} x^{0} \\
& x^{+} x^{-}-x^{-} x^{+}=\left(q^{1 / 2}-q^{-1 / 2}\right) x^{0} x^{0} .
\end{aligned}
$$

The algebra satisfied by derivatives is of the form

$$
\begin{aligned}
& \partial_{-} \partial_{0}=q^{-1} \partial_{0} \partial_{-}, \quad \partial_{0} \partial_{+}=q^{-1} \partial_{+} \partial_{0} \\
& \partial_{-} \partial_{+}-\partial_{+} \partial_{-}=\left(q^{1 / 2}-q^{-1 / 2}\right) \partial_{0} \partial_{0} .
\end{aligned}
$$

The action of derivatives on the coordinates is

$$
\begin{aligned}
& \partial_{-} x^{-}=1+q^{2} x^{-} \partial_{-}+\lambda q x^{0} \partial_{0}+\lambda(q-1) x^{+} \partial_{+}, \\
& \partial_{-} x^{0}=q x^{0} \partial_{-}-q^{1 / 2} \lambda x^{+} \partial_{0}, \quad \partial_{-} x^{+}=x^{+} \partial_{-}, \\
& \partial_{0} x^{-}=q x^{-} \partial_{0}-q^{1 / 2} \lambda x^{0} \partial_{+}, \\
& \partial_{0} x^{0}=1+q x^{0} \partial_{0}+q \lambda x^{+} \partial_{+}, \quad \partial_{0} x^{+}=q x^{+} \partial_{0}, \\
& \partial_{+} x^{-}=x^{-} \partial_{+}, \quad \partial_{+} x^{0}=q x^{0} \partial_{+}, \quad \partial_{+} x^{+}=1+q^{2} x^{+} \partial_{+} .
\end{aligned}
$$

It is convenient to introduce the dilatation operators

$$
\begin{aligned}
& \mu_{+}=1+q \lambda x^{+} \partial_{+} \\
& \Lambda=1+q \lambda \sum_{j=0, \pm} x^{j} \partial_{j}+q^{3} \lambda^{2}\left(q^{-1 / 2} x^{-} x^{+}+\frac{q}{1+q} x^{0} x^{0}\right)\left(q^{-1 / 2} \partial_{+} \partial_{-}+\frac{q}{1+q} \partial_{0} \partial_{0}\right) .
\end{aligned}
$$

The dilatation operators obey

$$
\begin{aligned}
& \mu_{+} x^{+}=q^{2} x^{+} \mu_{+}, \quad \mu_{+} \partial_{+}=q^{-2} \partial_{+} \mu_{+}, \\
& \Lambda x^{k}=q^{2} x^{k} \Lambda, \quad \Lambda \partial_{k}=q^{-2} \partial_{k} \Lambda, \quad \text { for } k=0, \pm .
\end{aligned}
$$

The real form $S O_{q}(3, R)$ (noted simply as $S O_{q}(3)$ whenever no confusion rised) of the noncommutative space $S O_{q}(3)$ is obtained by a consistent conjugation

$$
\begin{aligned}
& \overline{x^{i}}=C_{j i} x^{j} \\
& \overline{\partial_{i}}=-q^{-2} C_{i j} \Lambda^{-1}\left(q^{-1 / 2}\left[\partial_{+} \partial_{-}, x^{j}\right]+\frac{q}{1+q}\left[\partial_{0} \partial_{0}, x^{j}\right]\right),
\end{aligned}
$$


where the metric $C_{i j}$ is of the form

$$
C=\left(\begin{array}{lll} 
& & q^{-1 / 2} \\
& 1 & \\
q^{1 / 2} & &
\end{array}\right)
$$

It is should be noticed that throughout this paper we limit us at the case of $q$ being real.

By making use of the dilatation operators $\mu_{+}$and $\Lambda$, we introduce a new set of coordinates and derivatives

$$
\begin{aligned}
& \mathcal{X}^{-}=\Lambda^{-1 / 2} \mu_{+}^{-1 / 2}\left(x^{-}+q^{3 / 2} \lambda\left(q^{-1 / 2} x^{-} x^{+}+\frac{q}{1+q} x^{0} x^{0}\right) \partial_{+}\right) \\
& \mathcal{D}_{-}=q^{-1} \Lambda^{-1 / 2} \mu_{+}^{-1 / 2}\left(\partial_{-}+q^{3 / 2} \lambda\left(q^{-1 / 2} \partial_{+} \partial_{-}+\frac{q}{1+q} \partial_{0} \partial_{0}\right) x^{+}\right) \\
& \mathcal{X}^{0}=\mu_{+}^{-1 / 2} x^{0}, \quad \mathcal{D}_{0}=\mu_{+}^{-1 / 2} \partial_{0} \\
& \mathcal{X}^{+}=x^{+}, \quad \mathcal{D}_{+}=\partial_{+} .
\end{aligned}
$$

In terms of the new variables, the commutation relations among coordinates and derivatives of the noncommutative space $S O_{q}(3)$ are transformed as

$$
\begin{aligned}
& \mathcal{D}_{-} \mathcal{X}^{-}-\mathcal{X}^{-} \mathcal{D}_{-}=\mu_{-}^{-1}, \quad \mu_{-} \mathcal{X}^{-}=q^{2} \mathcal{X}^{-} \mu_{-}, \\
& \mu_{-} \mathcal{D}_{-}=q^{-2} \mathcal{D}_{-} \mu_{-}, \quad \mu_{-}^{-1} \equiv 1+\left(q^{-2}-1\right) \mathcal{X}^{-} \mathcal{D}_{-} ; \\
& \mathcal{D}_{0} \mathcal{X}^{0}-\mathcal{X}^{0} \mathcal{D}_{0}=\mu_{0}^{1 / 2}, \quad \mu_{0} \mathcal{X}^{0}=q^{2} \mathcal{X}^{0} \mu_{0} \\
& \mu_{0} \mathcal{D}_{0}=q^{-2} \mathcal{D}_{0} \mu_{0}, \quad \mu_{0}^{1 / 2} \equiv 1+(q-1) \mathcal{X}^{0} \mathcal{D}_{0} ; \\
& \mathcal{D}_{+} \mathcal{X}^{+}-\mathcal{X}^{+} \mathcal{D}_{+}=\mu_{+}, \quad \mu_{+} \mathcal{X}^{+}=q^{2} \mathcal{X}^{+} \mu_{+}, \\
& \mu_{+} \mathcal{D}_{+}=q^{-2} \mathcal{D}_{+} \mu_{+}, \quad \mu_{+}^{1 / 2}=1+\left(q^{2}-1\right) \mathcal{X}^{+} \mathcal{D}_{+} ; \\
& {\left[\mathcal{X}^{i}, \mathcal{X}^{j}\right]=0, \quad\left[\mathcal{D}_{i}, \mathcal{D}_{j}\right]=0, \quad\left[\mathcal{D}_{i}, \mathcal{X}_{j}\right]=0,} \\
& {\left[\mu_{i}, \mathcal{X}^{j}\right]=0, \quad\left[\mu_{i}, \mathcal{D}_{j}\right]=0, \quad \text { for } i \neq j .}
\end{aligned}
$$

The noncommutative surface $S_{q}^{3}$ in terms of the set of independent operators $\mathcal{X}^{j}$ and $\mathcal{D}_{j}$ is of the form

$$
\frac{q^{-1}}{1+q} \mathcal{X}^{0} \mathcal{X}^{0}+q^{-1 / 2} \Lambda^{1 / 2} \mu_{+}^{-1 / 2} \mathcal{X}^{+} \mathcal{X}^{-}=R^{2} .
$$

At the $q \rightarrow 1$ limit, $S_{q}^{3}$ reduces to the familiar $S^{3}$ surface with radius $R$.

The conjugate operation on $\mathcal{X}^{j}$ and $\mathcal{D}_{j}$ is induced by what on $x^{j}$ and $\partial_{j}$ (Eq.(7)), 


$$
\begin{aligned}
& \overline{\mathcal{X}}^{-}=\left(\bar{x}^{-}+q^{3 / 2} \lambda \bar{\partial}_{+}\left(q^{-1 / 2} \bar{x}^{+} \bar{x}^{-}+\frac{q}{1+q} \bar{x}^{0} \bar{x}^{0}\right)\right) \bar{\mu}_{+}{ }^{-1 / 2} \bar{\Lambda}^{-1 / 2}, \\
& \overline{\mathcal{D}}_{-}=q^{-1}\left(\bar{\partial}_{-}+q^{3 / 2} \lambda \bar{x}^{+}\left(q^{-1 / 2} \bar{\partial}_{-} \bar{\partial}_{+}-\frac{q}{1+q} \bar{\partial}_{0} \bar{\partial}_{0}\right)\right) \bar{\mu}_{+}{ }^{-1 / 2} \bar{\Lambda}^{-1 / 2}, \\
& \overline{\mathcal{X}}^{0}=\bar{x}^{0} \bar{\mu}_{+}^{-1 / 2}, \quad \overline{\mathcal{D}}_{0}=\bar{\partial}_{0} \bar{\mu}_{+}^{-1 / 2}, \\
& \overline{\mathcal{X}}^{+}=\bar{x}^{+}, \quad \overline{\mathcal{D}}_{+}=\bar{\partial}_{+} .
\end{aligned}
$$

Thus, we conclude that the Quantum Heisenberg-Weyl algebra corresponds to the noncommutative space $S O_{q}(3)$ can be decoupled into three independent subalgebras. And then, one can investigate properties of the noncommutative space by constructing Hilbert spaces of the three quantum subalgebras.

For the quantum algebra $\mathcal{A}_{-}$

$$
\begin{aligned}
& \mathcal{D}_{-} \mathcal{X}^{-}-\mathcal{X}^{-} \mathcal{D}_{-}=\mu_{-}^{-1}, \quad \mu_{-} \mathcal{X}^{-}=q^{2} \mathcal{X}^{-} \mu_{-}, \\
& \mu_{-} \mathcal{D}_{-}=q^{-2} \mathcal{D}_{-} \mu_{-}, \quad \mu_{-}^{-1} \equiv 1+\left(q^{-2}-1\right) \mathcal{X}^{-} \mathcal{D}_{-} \text {, }
\end{aligned}
$$

we construct a quantum coherent state $|z\rangle_{-}$as

$$
|z\rangle_{-}=\exp _{q^{2}}\left(-\frac{1}{2}\left|q^{-2} z\right|\right) \sum_{m=0}^{\infty} \frac{\left(-q^{-2} z\right)^{m}}{[m]_{q^{2}} !}\left(\mathcal{D}_{-}\right)^{m}|0\rangle_{-},
$$

where the notation of $q$-exponential

$$
\exp _{q}(x) \equiv \sum_{n=0}^{\infty} \frac{x^{n}}{[n]_{q} !}, \quad[n]_{q} !=[n]_{q}[n-1]_{q} \cdots[1]_{q}, \quad[n]_{q}=\frac{q^{n}-1}{q-1}
$$

has been used and the reference state $|0\rangle_{-}$was chosen such that $\mathcal{X}^{-}|0\rangle_{-}=0$.

As in the classical case, the coordinate $\mathcal{X}^{-}$is diagonal in the quantum coherent state basis

$$
\mathcal{X}^{-}|z\rangle_{-}=z|z\rangle_{-}
$$

The real value of parameter $z$ may be interpreted as position of an indispersive wavepocket[11]. Here we should notice that $z$ can be any complex number because of us working on a general quantum orthogonal space. The complex values of the quantum coherent state parameter are consistent with conjugate operation on the noncommutative space. 
Denote the quantum coherent state as

$$
|0, z\rangle_{-} \equiv|z\rangle_{-}
$$

we can construct a representation for the quantum algebra $\mathcal{A}_{-}$based on the quantum coherent state as

$$
\begin{aligned}
& \mathcal{X}^{-}|n, z\rangle_{-}=q^{2 n} z|n, z\rangle_{-}, \\
& \mathcal{D}_{-}|n, z\rangle_{-}=-q^{-1-2 n} \lambda^{-1} z^{-1}|n+1, z\rangle_{-}, \\
& \mu_{-}|n, z\rangle_{-}=|n-1, z\rangle_{-} .
\end{aligned}
$$

It is clear from the Hilbert space representation of the quantum algebra $\mathcal{A}_{-}$that the coordinates of the noncommutative orthogonal space is discretely latticed with exponentially increasing space distances. In fact, this is in agreement with the discrete difference representation of quantum derivatives,

$$
\mathcal{D} f(\mathcal{X})=\frac{f\left(q^{2} \mathcal{X}\right)-f(\mathcal{X})}{\left(q^{2}-1\right) \mathcal{X}}
$$

Similarly, we can construct the reference states $|0, z\rangle_{0}$ and $|0, z\rangle_{+}$as

$$
\begin{aligned}
& |0, z\rangle_{0}=\exp _{q^{-1}}\left(-\frac{1}{2}|q z|\right) \sum_{m=0}^{\infty} \frac{(-q z)^{m}}{[m]_{q^{-1}} !}\left(\mathcal{D}_{0}\right)^{m}|0\rangle_{0}, \quad \mathcal{X}^{0}|0\rangle_{0}=0 \\
& |0, z\rangle_{+}=\exp _{q^{-2}}\left(-\frac{1}{2}\left|q^{2} z\right|\right) \sum_{m=0}^{\infty} \frac{\left(-q^{2} z\right)^{m}}{[m]_{q^{-2}} !}\left(\mathcal{D}_{+}\right)^{m}|0\rangle_{+}, \quad \mathcal{X}^{+}|0\rangle_{+}=0 .
\end{aligned}
$$

The corresponding representations of the quantum algebras $\mathcal{A}_{0}$

$$
\begin{aligned}
& \mathcal{D}_{0} \mathcal{X}^{0}-\mathcal{X}^{0} \mathcal{D}_{0}=\mu_{0}^{1 / 2}, \quad \mu_{0} \mathcal{X}^{0}=q^{2} \mathcal{X}^{0} \mu_{0} \\
& \mu_{0} \mathcal{D}_{0}=q^{-2} \mathcal{D}_{0} \mu_{0}, \quad \mu_{0}^{1 / 2} \equiv 1+(q-1) \mathcal{X}^{0} \mathcal{D}_{0}
\end{aligned}
$$

and $\mathcal{A}_{+}$

$$
\begin{array}{lc}
\mathcal{D}_{+} \mathcal{X}^{+}-\mathcal{X}^{+} \mathcal{D}_{+}=\mu_{+}, & \mu_{+} \mathcal{X}^{+}=q^{2} \mathcal{X}^{+} \mu_{+} \\
\mu_{+} \mathcal{D}_{+}=q^{-2} \mathcal{D}_{+} \mu_{+}, & \mu_{+} \equiv 1+\left(q^{2}-1\right) \mathcal{X}^{+} \mathcal{D}_{+}
\end{array}
$$

are of the forms 


$$
\begin{aligned}
& \mathcal{X}^{0}|n, z\rangle_{0}=q^{n} z|n, z\rangle_{0} \\
& \mathcal{D}_{0}|n, z\rangle_{0}=\frac{q^{1-n}}{q-1} z^{-1}|n-1, z\rangle_{0} \\
& \mu_{0}|n, z\rangle_{0}=|n-1, z\rangle_{0}
\end{aligned}
$$

and

$$
\begin{aligned}
& \mathcal{X}^{+}|n, z\rangle_{+}=q^{2 n} z|n, z\rangle_{+}, \\
& \mathcal{D}_{+}|n, z\rangle_{+}=q^{1-2 n} \lambda^{-1} z^{-1}|n-1, z\rangle_{+}, \\
& \mu_{+}|n, z\rangle_{+}=|n-1, z\rangle_{+} .
\end{aligned}
$$

It has been strongly argued that the most direct and obvious physical cutoff of spacetime is from the formation of microscopic black holes as soon as too much energy would be accumulated into too small a region. Thus, from a physical point of view, the black holes should provide for a natural cutoff all by themselves. The cutoff distance scale is suspected to be the Planck scale. Because of this origin of spacetime cutoff, any geometry we working on there should be a same minimal cutoff $l_{p}$. For the classical geometry, the spacetime regularization is equal distance and thus there is one degree of freedom per Planck area. However, from the above discuss of noncommutative geometry, the spacetime is discretely latticed with exponentially increasing space distances. Thus, much less information can be stored in the noncommutative geometry. In fact, this may be the origin of the holography for quantum system of gravity.

For the noncommutative space $S O_{q}(3)$ with radius $R$, if the assumed minimal cutoff induced by the black holes themselves is the Planck scale $l_{p}$, it is not difficult to count the degrees of freedom $\mathcal{N}_{\text {bulk, }}$

$$
\begin{aligned}
\mathcal{N}_{\text {bulk }} & \approx \sum_{i=1}^{N} \frac{\left(q^{2 i} l_{p}\right)^{2}}{\left(q^{2 i} l_{p}-q^{2(i-1)} l_{p}\right)^{2}}, \quad q^{2 N}=R, \\
& =\frac{q^{4} \ln \left(\frac{R}{l_{p}}\right)}{2\left(q^{2}-1\right)^{2} \ln q} .
\end{aligned}
$$

By taking the deformation parameter of the noncommutative space value to be determined by the algebraic equation 


$$
q^{-4}\left(q^{2}-1\right)^{2} \ln q=\frac{\ln \left(\frac{R}{l_{p}}\right)}{8 \pi\left(\frac{R}{l_{p}}\right)^{2}},
$$

we can check that $\mathcal{N}_{\text {bulk }}$ is exact equal to the degrees of freedom on the classical limit of its boundary surface with radius $R, \mathcal{N}_{\text {boundary }}$

$$
\mathcal{N}_{\text {boundary }}=\frac{4 \pi R^{2}}{l_{p}^{2}} .
$$

And then one can write down the entropy of our world at Planck scale,

$$
S=4 \pi M^{2}+C
$$

where $M$ is the mass of the world (black hole) in natural units and $C$ a constant entropy can not determined. This just is what was called holography or dimension reduction in quantum theory of gravity by 't Hooft. At Planck scale, our world is not $3+1$ dimensional. Rather, the observable degrees of freedom can best be described as if they were Boolean variables defined on a 2 dimensional lattice, evolving with time. It is clear now that the exact meaning of the holography can interpreted as that the quantum theory of gravity in higher dimensional noncommutative space is equivalent to the theory living on the classical limit of spacetime boundary. This supplies a reasonable picture for the 't Hooft's holography.

\section{Noncommutative $\mathrm{AdS}_{2 n}$ space and exponential reg- ularization}

The noncommutative $\mathrm{AdS}_{2 n}$ space can be defined as the $2 n$-dimensional noncommutative real hyperboloid embedded in a $(2 n+1)$-dimensional space with coordinates $x^{i}$ $(i=-n,-n+1, \cdots,-1,0,1, \cdots, n)$

$$
\begin{aligned}
& \frac{1}{1+q^{2 n-1}} C_{i j} x^{i} x^{j}=-\frac{1}{a^{2}}, \\
& \bar{x}^{i}=C_{j i} M_{j k} x^{k},
\end{aligned}
$$


where $\rho_{-i}=i-\frac{1}{2}, \rho_{0}=0, \rho_{i}=-i+\frac{1}{2}$, and the metric $C_{i j}=q^{-\rho_{i}} \delta_{i,-j}$ and

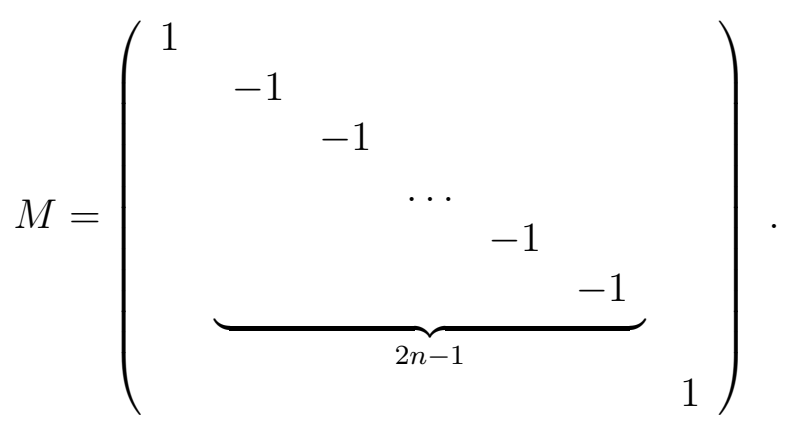

It is easy to check that, at the $q \longrightarrow 1$ limit, the noncommutative $\mathrm{AdS}_{2 n}$ space deduces to the familiar $\mathrm{AdS}_{2 n}$ space

$$
\eta_{a b} x^{a} x^{b}=-\frac{1}{a^{2}}
$$

in $R^{2 n+1}$ with Cartesian coordinates $x^{a}$, where $\eta_{a b}=\operatorname{diag}(-1, \underbrace{1, \cdots, 1}_{2 n-1},-1)$.

For the noncommutative $\mathrm{AdS}_{2 n}$ space, in components, the commutation relations among coordinates are

$$
\begin{aligned}
x^{i} x^{j} & =q x^{j} x^{i}, \quad \text { for } i<j \text { and } i \neq-j, \\
x^{i} x^{-i} & =x^{-i} x^{i}+\lambda q^{i-3 / 2} L_{i-1} \\
& =q^{-2} x^{-i} x^{i}+\lambda q^{i-3 / 2} L_{i}, \quad \text { for } i>0,
\end{aligned}
$$

where we have used the notation for intermediate lengths

$$
L_{i}=\sum_{k=1}^{i} q^{\rho_{k}} x^{-k} x^{k}+\frac{q}{1+q} x^{0} x^{0} .
$$

By making use of the intermediate Laplacians

$$
\Delta_{i}=\sum_{k=1}^{i} q^{\rho_{k}} \partial_{k} \partial_{-k}+\frac{q}{1+q} \partial_{0} \partial_{0}
$$

the algebra satisfied by the derivatives can be written compactly as

$$
\begin{aligned}
\partial_{i} \partial_{j} & =q^{-1} \partial_{j} \partial_{i}, \quad \text { for } i<j \text { and } i \neq-j, \\
\partial_{-i} \partial_{i} & =\partial_{i} \partial_{-i}+\lambda q^{i-3 / 2} \Delta_{i-1} \\
& =q^{-2} \partial_{i} \partial_{-i}+\lambda q^{i-3 / 2} \Delta_{i}, \quad \text { for } i>0 .
\end{aligned}
$$

The commutation relations among the coordinates and derivatives are as follows 


$$
\begin{aligned}
& \partial_{-i} x^{i}=x^{i} \partial_{-i}, \quad \text { for } i \neq 0, \\
& \partial_{i} x^{j}=q x^{j} \partial_{i}, \quad \text { for } j>-i, \text { and } j \neq i, \\
& \partial_{i} x^{j}=q x^{j} \partial_{i}-q \lambda q^{-\rho_{j}-\rho_{k}} x^{-i} \partial_{-j}, \quad \text { for } j<-i, \quad \text { and } i \neq j, \\
& \partial_{j} x^{j}=1+q^{2} x^{j} \partial_{j}+q \lambda \sum_{k>j} x^{k} \partial_{k}-q^{1-2 \rho_{j}} \lambda x^{-j} \partial_{-j}, \quad \text { for } j<0, \\
& \partial_{0} x^{0}=1+q x^{0} \partial_{0}+q \lambda \sum_{k>0}^{k} x^{k} \partial_{k}, \\
& \partial_{j} x^{j}=1+q^{2} x^{j} \partial_{j}+q \lambda \sum_{k>j}^{k} x^{k} \partial_{k}, \quad \text { for } j>0 .
\end{aligned}
$$

For convenient, we introduce the dilatation operator $\Lambda_{m}(0<m \leq n)$ as

$$
\Lambda_{m}=1+q \lambda E_{m}+q^{2 m+1} \lambda^{2} L_{m} \Delta_{m}, \quad E_{m}=\sum_{j=-m}^{m} x^{j} \partial_{j} .
$$

The dilatation operator satisfies

$$
\Lambda_{m} x^{k}=q^{2} x^{k} \Lambda_{m}, \quad \Lambda_{m} \partial_{k}=q^{-2} \partial_{k} \Lambda_{m}, \quad \text { for }|k| \leq m
$$

The noncommutative $\mathrm{AdS}_{2 n}$ space is accompanied with the conjugation [12]

$$
\begin{aligned}
& \bar{x}^{i}=C_{j i} M_{j k} x^{k}, \\
& \bar{\partial}_{i}=-q^{-2} \Lambda_{n}^{-1} C_{i j} M_{j k}\left[\Delta_{n}, x^{k}\right] .
\end{aligned}
$$

For $k>0$, using the notations

$$
\begin{aligned}
& y^{-k}=x^{-k}+q^{k+1 / 2} \lambda L_{k} \partial_{+k}, \\
& \delta_{-k}=\partial_{-k}+q^{k+1 / 2} \lambda \Delta_{k} x^{+k},
\end{aligned}
$$

we can construct a set of independent basis 13 on the noncommutative $\mathrm{AdS}_{2 n}$ space,

$$
\begin{aligned}
& \mathcal{X}^{n}=x^{n}, \\
& \mathcal{D}_{n}=\partial_{n}, \\
& \mathcal{X}^{+j}=\mu_{n}^{-1 / 2} \mu_{n-1}^{-1 / 2} \cdots \mu_{j+1}^{-1 / 2} x^{+j}, \quad \text { for } n>j \geq 0, \\
& \mathcal{D}_{+j}=\mu_{n}^{-1 / 2} \mu_{n-1}^{-1 / 2} \cdots \mu_{j+1}^{-1 / 2} \partial_{+j}, \\
& \mathcal{X}^{-j}=\mu_{n}^{-1 / 2} \mu_{n-1}^{-1 / 2} \cdots \mu_{j+1}^{-1 / 2} \Lambda_{+j}^{-1 / 2} \mu_{+j}^{-1 / 2} y^{-j}, \\
& \mathcal{D}_{-j}=q^{-1} \mu_{n}^{-1 / 2} \mu_{n-1}^{-1 / 2} \cdots \mu_{j+1}^{-1 / 2} \Lambda_{+j}^{-1 / 2} \mu_{+j}^{-1 / 2} \delta_{-j}, \\
& \mathcal{X}^{-n}=\Lambda_{+n}^{-1 / 2} \mu_{+n}^{-1 / 2} y^{-n}, \\
& \mathcal{D}_{-j}=q^{-1} \Lambda_{+n}^{-1 / 2} \mu_{+n}^{-1 / 2} \delta_{-n},
\end{aligned}
$$


where $\left(\mu_{ \pm i}\right)^{ \pm 1}=\mathcal{D}_{ \pm i} \mathcal{X}^{ \pm i}-\mathcal{X}^{ \pm i} \mathcal{D}_{ \pm i}$ and $\mu_{0}^{1 / 2}=\mathcal{D}_{0} \mathcal{X}^{0}-\mathcal{X}^{0} \mathcal{D}_{0}$

We note that the $\mu_{i}$ 's satisfy simple commutation relations with the new variables $\mathcal{X}^{j}$ and $\mathcal{D}_{j}$,

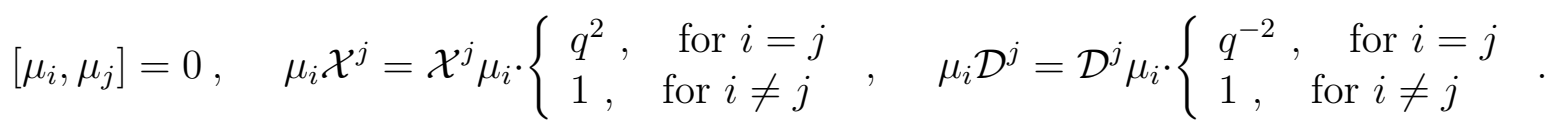

For the new basis of coordinates and derivatives on the noncommutative $\mathrm{AdS}_{2 n}$ space, it is not difficult to show that

$$
\begin{aligned}
& \mathcal{D}_{-k} \mathcal{X}^{-k}=1+q^{-2} \mathcal{X}^{-k} \mathcal{D}_{-k}, \quad \text { for } k>0 \\
& \mathcal{D}_{0} \mathcal{X}^{0}=1+q \mathcal{X}^{0} \mathcal{D}_{0}, \\
& \mathcal{D}_{+k} \mathcal{X}^{+k}=1+q^{2} \mathcal{X}^{+k} \mathcal{D}_{+k} \\
& {\left[\mathcal{D}_{i}, \mathcal{D}_{j}\right]=0, \quad\left[\mathcal{X}^{i}, \mathcal{X}^{j}\right]=0} \\
& \mathcal{D}_{i} \mathcal{X}^{j}=\mathcal{X}^{j} \mathcal{D}_{i}, \quad \text { for } i \neq j .
\end{aligned}
$$

The noncommutative $\mathrm{AdS}_{2 n}$ space in terms of the $\mathcal{X}^{i}$ and $\mathcal{D}_{i}$ is of the form

$$
\sum_{j=1}^{n} q^{\rho_{j}-2(n-j)} \Lambda_{j}^{1 / 2} \mu_{j}^{-1 / 2} \mathcal{X}^{j} \mathcal{X}^{-j}+\frac{q^{-2 n+1}}{1+q} \mathcal{X}^{0} \mathcal{X}^{0}=-\frac{1}{a^{2}}
$$

The conjugate operation on the independent set of operators $\mathcal{X}^{j}$ and $\mathcal{D}_{j}$ is induced from what on $x^{i}$ and $\partial_{i}$ (Eq.(30)),

$$
\begin{aligned}
& \overline{\mathcal{X}}^{n}=\bar{x}^{n}, \\
& \overline{\mathcal{D}}_{n}=\bar{\partial}_{n}, \\
& \overline{\mathcal{X}}^{+j}=\bar{x}^{+j} \bar{\mu}_{j+1}{ }^{-1 / 2} \bar{\mu}_{j+2}{ }^{-1 / 2} \cdots \bar{\mu}_{n}^{-1 / 2}, \quad \text { for } n>j \geq 0, \\
& \overline{\mathcal{D}}_{+j}=\bar{\partial}_{+j} \bar{\mu}_{j+1}{ }^{-1 / 2} \bar{\mu}_{j+2}{ }^{-1 / 2} \cdots \bar{\mu}_{n}{ }^{-1 / 2}, \\
& \overline{\mathcal{X}}^{-j}=\bar{y}^{-j} \bar{\mu}_{+j}{ }^{-1 / 2} \bar{\Lambda}_{+j}{ }^{-1 / 2} \bar{\mu}_{j+1}{ }^{-1 / 2} \bar{\mu}_{j+2}{ }^{-1 / 2} \cdots \bar{\mu}_{n}{ }^{-1 / 2}, \\
& \overline{\mathcal{D}}_{-j}=q^{-1} \bar{\delta}_{-j} \bar{\mu}_{+j}{ }^{-1 / 2} \bar{\Lambda}_{+j}{ }^{-1 / 2} \bar{\mu}_{j+1}{ }^{-1 / 2} \bar{\mu}_{j+2}{ }^{-1 / 2} \cdots \bar{\mu}_{n}{ }^{-1 / 2}, \\
& \overline{\mathcal{X}}^{-n}=\bar{y}^{-n} \bar{\mu}_{+n}{ }^{-1 / 2} \bar{\Lambda}_{+n}{ }^{-1 / 2}, \\
& \overline{\mathcal{D}}_{-j}=q^{-1} \bar{\delta}_{-n} \bar{\mu}_{+n}{ }^{-1 / 2} \bar{\Lambda}_{+n}{ }^{-1 / 2} .
\end{aligned}
$$

Thus, the Quantum Heisenberg-Weyl algebra corresponds to the noncommutative $\mathrm{AdS}_{2 n}$ space can be decoupled into $(2 n+1)$ independent subalgebras.

For the quantum algebra $\mathcal{A}_{-k}(n \geq k>0)$ 


$$
\begin{aligned}
& \mathcal{D}_{-k} \mathcal{X}^{-k}-\mathcal{X}^{-k} \mathcal{D}_{-k}=\mu_{-k}^{-1}, \quad \mu_{-k} \mathcal{X}^{-k}=q^{2} \mathcal{X}^{-k} \mu_{-k} \\
& \mu_{-} \mathcal{D}_{-k}=q^{-2} \mathcal{D}_{-k} \mu_{-k}, \quad \mu_{-}^{-1} \equiv 1+\left(q^{-2}-1\right) \mathcal{X}^{-k} \mathcal{D}_{-k}
\end{aligned}
$$

we construct the quantum coherent state $|0, z\rangle_{-k}$ as

$$
\begin{aligned}
& |0, z\rangle_{-k}=\exp _{q^{2}}\left(-\frac{1}{2}\left|q^{-2} z\right|\right) \sum_{m=0}^{\infty} \frac{\left(-q^{-2} z\right)^{m}}{[m]_{q^{2}} !}\left(\mathcal{D}_{-k}\right)^{m}|0\rangle_{-k}, \\
& \mathcal{X}^{-k}|0, z\rangle_{-k}=z|0, z\rangle_{-k},
\end{aligned}
$$

where the reference state $|0\rangle_{-k}$ was chosen such that $\mathcal{X}^{-k}|0\rangle_{-k}=0$.

From the coherent state $|0, z\rangle_{-k}$, we can construct a representation for the quantum algebra $\mathcal{A}_{-k}$ as

$$
\begin{aligned}
& \mathcal{X}^{-k}|n, z\rangle_{-}=q^{2 n} z|n, z\rangle_{-k}, \\
& \mathcal{D}_{-k}|n, z\rangle_{-}=-q^{-1-2 n} \lambda^{-1} z^{-1}|n+1, z\rangle_{-k}, \\
& \mu_{-k}|n, z\rangle_{-}=|n-1, z\rangle_{-k} .
\end{aligned}
$$

The quantum coherent state corresponds to the quantum algebras $\mathcal{A}_{0}$

$$
\begin{aligned}
& \mathcal{D}_{0} \mathcal{X}^{0}-\mathcal{X}^{0} \mathcal{D}_{0}=\mu_{0}^{1 / 2}, \quad \mu_{0} \mathcal{X}^{0}=q^{2} \mathcal{X}^{0} \mu_{0}, \\
& \mu_{0} \mathcal{D}_{0}=q^{-2} \mathcal{D}_{0} \mu_{0}, \quad \mu_{0}^{1 / 2} \equiv 1+(q-1) \mathcal{X}^{0} \mathcal{D}_{0}
\end{aligned}
$$

is

$$
\begin{aligned}
& |0, z\rangle_{0}=\exp _{q^{-1}}\left(-\frac{1}{2}|q z|\right) \sum_{m=0}^{\infty} \frac{(-q z)^{m}}{[m]_{q^{-1}} !}\left(\mathcal{D}_{0}\right)^{m}|0\rangle_{0}, \quad \mathcal{X}^{0}|0\rangle_{0}=0 \\
& \mathcal{X}^{0}|0, z\rangle_{0}=z|0, z\rangle_{0}
\end{aligned}
$$

Representation of the quantum algebra $\mathcal{A}_{0}$ based on the quantum coherent state $|0, z\rangle_{0}$ is as

$$
\begin{aligned}
& \mathcal{X}^{0}|n, z\rangle_{0}=q^{n} z|n, z\rangle_{0}, \\
& \mathcal{D}_{0}|n, z\rangle_{0}=\frac{q^{1-n}}{q-1} z^{-1}|n-1, z\rangle_{0}, \\
& \mu_{0}|n, z\rangle_{0}=|n-1, z\rangle_{0} .
\end{aligned}
$$

The quantum coherent state corresponds to the quantum algebra $\mathcal{A}_{+k}$

$$
\begin{aligned}
& \mathcal{D}_{+k} \mathcal{X}^{+k}-\mathcal{X}^{+k} \mathcal{D}_{+k}=\mu_{+k}, \quad \mu_{+k} \mathcal{X}^{+k}=q^{2} \mathcal{X}^{+k} \mu_{+k} \\
& \mu_{+k} \mathcal{D}_{+k}=q^{-2} \mathcal{D}_{+k} \mu_{+k}, \quad \mu_{+k} \equiv 1+\left(q^{2}-1\right) \mathcal{X}^{+k} \mathcal{D}_{+k}
\end{aligned}
$$

is of the form 


$$
\begin{aligned}
& |0, z\rangle_{+k}=\exp _{q^{-2}}\left(-\frac{1}{2}\left|q^{2} z\right|\right) \sum_{m=0}^{\infty} \frac{\left(-q^{2} z\right)^{m}}{[m]_{q^{-2}} !}\left(\mathcal{D}_{+k}\right)^{m}|0\rangle_{+k}, \quad \mathcal{X}^{+k}|0\rangle_{+k}=0, \\
& \mathcal{X}^{+k}|0, z\rangle_{+k}=z|0, z\rangle_{+k} .
\end{aligned}
$$

Then, by writing down the representation of the quantum algebra $\mathcal{A}_{+k}$

$$
\begin{aligned}
& \mathcal{X}^{+k}|n, z\rangle_{+k}=q^{2 n} z|n, z\rangle_{+k}, \\
& \mathcal{D}_{+k}|n, z\rangle_{+k}=q^{1-2 n} \lambda^{-1} z^{-1}|n-1, z\rangle_{+k}, \\
& \Lambda_{+k}|n, z\rangle_{+k}=|n-1, z\rangle_{+k},
\end{aligned}
$$

we complete the Hilbert space constructure of the noncommutative $\operatorname{AdS}_{2 n}$ space. This shows that the noncommutative $\mathrm{AdS}_{2 n}$ space is discretely latticed with exponentially increasing space distances. The minimal cutoff induced by the quantum gravity itself is the Planck scale $l_{p}$. As in the case of noncommutative orthogonal space, the exponential regularization may effectively reduce the amount of observable degrees of freedom of the noncommutative $\mathrm{AdS}_{2 n}$ space, even one can not enumerate it exactly because of being infinite [14. An adequately adopted noncommutative deformation parameter $q$ (it may be even more closer to 1 than the case of limited geometry) can equate the entropy of the quantum system of gravity in the bulk of noncommutative $\mathrm{AdS}_{2 n}$ space and what on the classical limit of its boundary. The commutative boundary is equal distance regularized and possesses conformally invariant symmetry. This is crucial for the AdS/CFT correspondence.

\section{Noncommutative $\mathrm{AdS}_{2 n-1}$ space with exponential regularization}

For the noncommutative $\mathrm{AdS}_{2 n-1}$ space, commutation relations among coordinates $x^{i}$ $(i=-n,-n+1, \cdots,-2,-1,+1,+2, \cdots, n)$ are

$$
\begin{aligned}
x^{i} x^{j} & =q x^{j} x^{i}, \quad \text { for } i<j \text { and } i \neq-j, \\
x^{i} x^{-i} & =x^{-i} x^{i}+\lambda q^{i-2} L_{i-1} \\
& =q^{-2} x^{-i} x^{i}+\lambda q^{i-2} L_{i}, \quad \text { for } i>0,
\end{aligned}
$$


where we have used the notation for intermediate lengths

$$
L_{i}=\sum_{k=1}^{i} q^{\rho_{k}} x^{-k} x^{k}, \quad \rho_{-k}=k-1, \quad \rho_{k}=-k+1 .
$$

By making use of the intermediate Laplacians

$$
\Delta_{i}=\sum_{k=1}^{i} q^{\rho_{k}} \partial_{k} \partial_{-k}
$$

the algebra satisfied by the derivatives can be written compactly as

$$
\begin{aligned}
\partial_{i} \partial_{j} & =q^{-1} \partial_{j} \partial_{i}, \quad \text { for } i<j \text { and } i \neq-j, \\
\partial_{-i} \partial_{i} & =\partial_{i} \partial_{-i}+\lambda q^{i-2} \Delta_{i-1} \\
& =q^{-2} \partial_{i} \partial_{-i}+\lambda q^{i-2} \Delta_{i}, \quad \text { for } i>0
\end{aligned}
$$

The commutation relations among the coordinates and derivatives are as follows

$$
\begin{aligned}
& \partial_{-i} x^{i}=x^{i} \partial_{-i}, \quad \text { for } i \neq 0, \\
& \partial_{i} x^{j}=q x^{j} \partial_{i}, \quad \text { for } j>-i, \text { and } j \neq i, \\
& \partial_{i} x^{j}=q x^{j} \partial_{i}-q \lambda q^{-\rho_{j}-\rho_{k}} x^{-i} \partial_{-j}, \quad \text { for } j<-i, \quad \text { and } i \neq j, \\
& \partial_{j} x^{j}=1+q^{2} x^{j} \partial_{j}+q \lambda \sum_{k>j} x^{k} \partial_{k}-q^{1-2 \rho_{j}} \lambda x^{-j} \partial_{-j}, \quad \text { for } j<0, \\
& \partial_{j} x^{j}=1+q^{2} x^{j} \partial_{j}+q \lambda \sum_{k>j} x^{k} \partial_{k}, \quad \text { for } j>0 .
\end{aligned}
$$

For convenient, we introduce the dilatation operator $\Lambda_{m}(0<m \leq n)$ as

$$
\Lambda_{m}=1+q \lambda E_{m}+q^{2 m} \lambda^{2}\left(1+q^{2 n-2}\right) L_{m} \Delta_{m}, \quad E_{m}=\sum_{j=-m}^{m} x^{j} \partial_{j} .
$$

The dilatation operator satisfies

$$
\Lambda_{m} x^{k}=q^{2} x^{k} \Lambda_{m}, \quad \Lambda_{m} \partial_{k}=q^{-2} \partial_{k} \Lambda_{m}, \quad \text { for }<0|k| \leq m
$$

The noncommutative $\mathrm{AdS}_{2 n-1}$ space is accompanied with the conjugation

$$
\begin{aligned}
& \bar{x}^{i}=C_{j i} N_{j k} x^{k}, \\
& \bar{\partial}_{i}=-q^{-2} \Lambda_{n}^{-1} C_{i j} N_{j k}\left[\Delta_{n}, x^{k}\right],
\end{aligned}
$$


where

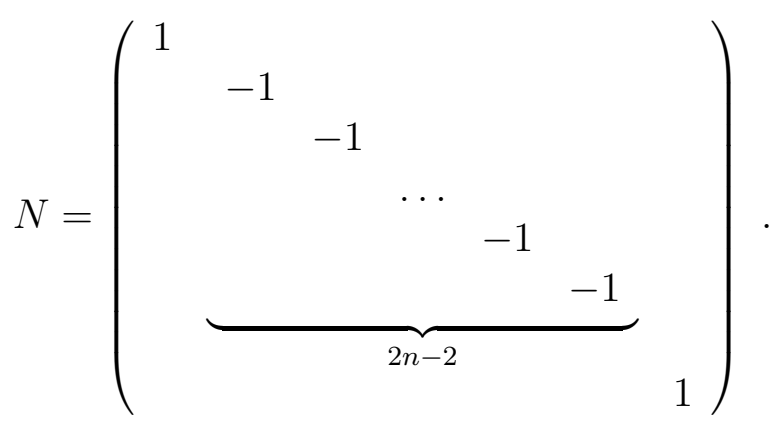

For $k>0$, using the notations

$$
\begin{aligned}
& y^{-k}=x^{-k}+q^{k+1 / 2} \lambda\left(1+q^{2 n-2}\right) L_{k} \partial_{+k}, \\
& \delta_{-k}=\partial_{-k}+q^{k+1 / 2} \lambda \Delta_{k} x^{+k}
\end{aligned}
$$

we can construct a new set of variables for the coordinates and derivatives,

$$
\begin{aligned}
& \mathcal{X}^{n}=x^{n}, \\
& \mathcal{D}_{n}=\partial_{n}, \\
& \mathcal{X}^{+j}=\mu_{n}^{-1 / 2} \mu_{n-1}^{-1 / 2} \cdots \mu_{j+1}^{-1 / 2} x^{+j}, \quad \text { for } n>j>0, \\
& \mathcal{D}_{+j}=\mu_{n}^{-1 / 2} \mu_{n-1 / 2}^{-1} \cdots \mu_{j+1}^{-1 / 2} \partial_{+j}, \\
& \mathcal{X}^{-j}=\mu_{n}^{-1 / 2} \mu_{n-1}^{-1 / 2} \cdots \mu_{j+1}^{-1 / 2} \Lambda_{+j}^{-1 / 2} \mu_{+j}^{-1 / 2} y^{-j}, \\
& \mathcal{D}_{-j}=q^{-1} \mu_{n}^{-1 / 2} \mu_{n-1}^{-1 / 2} \cdots \mu_{j+1}^{-1 / 2} \Lambda_{+j}^{-1 / 2} \mu_{+j}^{-1 / 2} \delta_{-j}, \\
& \mathcal{X}^{-n}=\Lambda_{+n}^{-1 / 2} \mu_{+n}^{-1 / 2} y^{-n}, \\
& \mathcal{D}_{-j}=q^{-1} \Lambda_{+n}^{-1 / 2} \mu_{+n}^{-1 / 2} \delta_{-n},
\end{aligned}
$$

where $\left(\mu_{ \pm i}\right)^{ \pm 1}=\mathcal{D}_{ \pm i} \mathcal{X}^{ \pm i}-\mathcal{X}^{ \pm i} \mathcal{D}_{ \pm i}$.

Commutation relations among the new basis of coordinates and derivatives on the noncommutative $\mathrm{AdS}_{2 n-1}$ space are

$$
\begin{aligned}
& \mathcal{D}_{-k} \mathcal{X}^{-k}=1+q^{-2} \mathcal{X}^{-k} \mathcal{D}_{-k}, \quad \text { for } k>0 \\
& \mathcal{D}_{+k} \mathcal{X}^{+k}=1+q^{2} \mathcal{X}^{+k} \mathcal{D}_{+k}, \\
& {\left[\mathcal{D}_{i}, \mathcal{D}_{j}\right]=0, \quad\left[\mathcal{X}^{i}, \mathcal{X}^{j}\right]=0} \\
& \mathcal{D}_{i} \mathcal{X}^{j}=\mathcal{X}^{j} \mathcal{D}_{i}, \quad \text { for } i \neq j .
\end{aligned}
$$

The noncommutative $\mathrm{AdS}_{2 n-1}$ space in terms of the $\mathcal{X}^{i}$ and $\mathcal{D}_{i}$ is of the form

$$
\sum_{j=1}^{n} q^{\rho_{j}-2(n-j)} \Lambda_{j}^{1 / 2} \mu_{j}^{-1 / 2} \mathcal{X}^{j} \mathcal{X}^{-j}=-\frac{1}{a^{2}}
$$

The conjugate operation on the operators $\mathcal{X}^{j}$ and $\mathcal{D}_{j}$ is induced by the conjugation on $x^{i}$ and $\partial_{i}$, 


$$
\begin{aligned}
& \overline{\mathcal{X}}^{n}=\bar{x}^{n}, \\
& \overline{\mathcal{D}}_{n}=\bar{\partial}_{n}, \\
& \overline{\mathcal{X}}^{+j}=\bar{x}^{+j} \bar{\mu}_{j+1}{ }^{-1 / 2} \bar{\mu}_{j+2}{ }^{-1 / 2} \cdots \bar{\mu}_{n}{ }^{-1 / 2}, \quad \text { for } n>j>0, \\
& \overline{\mathcal{D}}_{+j}=\bar{\partial}_{+j} \bar{\mu}_{j+1}{ }^{-1 / 2} \bar{\mu}_{j+2}{ }^{-1 / 2} \cdots \bar{\mu}_{n}{ }^{-1 / 2}, \\
& \overline{\mathcal{X}}^{-j}=\bar{y}^{-j} \bar{\mu}_{+j}{ }^{-1 / 2} \bar{\Lambda}_{+j}{ }^{-1 / 2} \bar{\mu}_{j+1}{ }^{-1 / 2} \bar{\mu}_{j+2}{ }^{-1 / 2} \cdots \bar{\mu}_{n}{ }^{-1 / 2}, \\
& \overline{\mathcal{D}}_{-j}=q^{-1} \bar{\delta}_{-j} \bar{\mu}_{+j}{ }^{-1 / 2} \bar{\Lambda}_{+j}{ }^{-1 / 2} \bar{\mu}_{j+1}{ }^{-1 / 2} \bar{\mu}_{j+2}{ }^{-1 / 2} \cdots \bar{\mu}_{n}{ }^{-1 / 2}, \\
& \overline{\mathcal{X}}^{-n}=\bar{y}^{-n} \bar{\mu}_{+n}{ }^{-1 / 2} \bar{\Lambda}_{+n}{ }^{-1 / 2}{ }^{-1 / 2} . \\
& \overline{\mathcal{D}}_{-j}=q^{-1} \bar{\delta}_{-n} \bar{\mu}_{+n}{ }^{-1 / 2} \bar{\Lambda}_{+n}{ }^{-1 / 2} .
\end{aligned}
$$

Then, the Quantum Heisenberg-Weyl algebra corresponds to the noncommutative $\mathrm{AdS}_{2 n-1}$ space is decoupled into $2 n$ independent subalgebras.

For the quantum algebra $\mathcal{A}_{-k}(n \geq k>0)$

$$
\begin{aligned}
& \mathcal{D}_{-k} \mathcal{X}^{-k}-\mathcal{X}^{-k} \mathcal{D}_{-k}=\mu_{-k}^{-1}, \quad \mu_{-k} \mathcal{X}^{-k}=q^{2} \mathcal{X}^{-k} \mu_{-k}, \\
& \mu_{-} \mathcal{D}_{-k}=q^{-2} \mathcal{D}_{-k} \mu_{-k}, \quad \mu_{-}^{-1} \equiv 1+\left(q^{-2}-1\right) \mathcal{X}^{-k} \mathcal{D}_{-k}
\end{aligned}
$$

we have the quantum coherent state $|0, z\rangle_{-k}$ as

$$
\begin{aligned}
& |0, z\rangle_{-k}=\exp _{q^{2}}\left(-\frac{1}{2}\left|q^{-2} z\right|\right) \sum_{m=0}^{\infty} \frac{\left(-q^{-2} z\right)^{m}}{[m]_{q^{2}} !}\left(\mathcal{D}_{-k}\right)^{m}|0\rangle_{-k}, \quad \mathcal{X}^{-k}|0\rangle_{-k}=0 \\
& \mathcal{X}^{-k}|0, z\rangle_{-k}=z|0, z\rangle_{-k} .
\end{aligned}
$$

From the coherent state $|0, z\rangle_{-k}$, we can construct a representation for the quantum algebra $\mathcal{A}_{-k}$ as

$$
\begin{aligned}
& \mathcal{X}^{-k}|n, z\rangle_{-}=q^{2 n} z|n, z\rangle_{-k} \\
& \mathcal{D}_{-k}|n, z\rangle_{-}=-q^{-1-2 n} \lambda^{-1} z^{-1}|n+1, z\rangle_{-k}, \\
& \mu_{-k}|n, z\rangle_{-}=|n-1, z\rangle_{-k} .
\end{aligned}
$$

Similarly, we can construct reference state $|0, z\rangle_{+k}(n \geq k>0)$ as

$$
\begin{aligned}
& |0, z\rangle_{+k}=\exp _{q^{-2}}\left(-\frac{1}{2}\left|q^{2} z\right|\right) \sum_{m=0}^{\infty} \frac{\left(-q^{2} z\right)^{m}}{[m]_{q^{-2}} !}\left(\mathcal{D}_{+k}\right)^{m}|0\rangle_{+k}, \quad \mathcal{X}^{+k}|0\rangle_{+k}=0 \\
& \mathcal{X}^{+k}|0, z\rangle_{+k}=z|0, z\rangle_{+k} .
\end{aligned}
$$

The corresponding representation of the quantum algebras $\mathcal{A}_{+k}$

$$
\begin{aligned}
& \mathcal{D}_{+k} \mathcal{X}^{+k}-\mathcal{X}^{+k} \mathcal{D}_{+k}=\mu_{+k}, \quad \mu_{+k} \mathcal{X}^{+k}=q^{2} \mathcal{X}^{+k} \mu_{+k} \\
& \mu_{+k} \mathcal{D}_{+k}=q^{-2} \mathcal{D}_{+k} \mu_{+k}, \quad \mu_{+k} \equiv 1+\left(q^{2}-1\right) \mathcal{X}^{+k} \mathcal{D}_{+k}
\end{aligned}
$$


is of the form

$$
\begin{aligned}
& \mathcal{X}^{+k}|n, z\rangle_{+k}=q^{2 n} z|n, z\rangle_{+k}, \\
& \mathcal{D}_{+k}|n, z\rangle_{+k}=q^{1-2 n} \lambda^{-1} z^{-1}|n-1, z\rangle_{+k}, \\
& \Lambda_{+k}|n, z\rangle_{+k}=|n-1, z\rangle_{+k} .
\end{aligned}
$$

As the noncommutative $\mathrm{AdS}_{2 n}$ space did, the noncommutative $\operatorname{AdS}_{2 n-1}$ space is also discretely latticed with exponentially increasing space distances. The minimal cutoff induced by the quantum gravity itself is the Planck scale $l_{p}$. The exponential regularization effectively reduces degrees of freedom in the noncommutative $\operatorname{AdS}_{2 n-1}$ space. A very small amount of displacement of the noncommutative deformation parameter $q$ from unity equates the entropy of the quantum theory of gravity in the bulk of noncommutative $\mathrm{AdS}_{2 n-1}$ space and what on the commutative limit of its boundary surface. The commutative boundary is equal distance regularized and possesses a conformally invariant symmetry. Thus, the equivalent theory living on the spacetime boundary of the quantum system of gravity on the background of noncommutative AdS space is a conformal field theory. This is the bases for the AdS/CFT correspondence.

\section{Concluding remarks}

In this paper, by constructing Hilbert space with quantum coherent state as reference one, we have presented a kind of special regularization with exponentially increasing spacetime cutoff for both orthogonal and AdS space based on noncommutative geometry. It has been used to argue that the same minimal cutoff of any geometry is the Planck scale $l_{p}$, that the most direct and obvious physical cutoff is from the formation of microscopic black holes as soon as too much energy would be accumulated into too small a region. We have obtained results which show a very small $\left(<10^{-15}\right)$ displacement of the noncommutative deformation parameter from its classical value (1) reduces sharply the entropy of quantum system of gravity. The noncommutative deformation parameter was determined by a well-defined algebraic equation. The noncommutative space $S O_{q}(3)$ with such a deformation parameter have the same entropy 
or degrees of freedom with classical $S^{3}$ surface. This is just the so called holography for quantum theory of gravity. The holography makes the quantum theory of gravity on the noncommutative $\mathrm{AdS}_{d}$ space equivalent to the conformally invariant quantum field theory living on the classical limit of its boundary. This is the bases of the AdS/CFT correspondence of string theory and M theory. Here we should stress that the proper geometry for quantum gravity may be noncommutative but not classical continuum geometry. This is in agreement with the long time suspecting that the small scale structure of spacetime might not be adequately described by classical continuum geometry and the noncommutative spacetime might be a realistic picture of how spacetime behaves near the Planck scale. Strong quantum fluctuations of gravity at this spacetime scale may make points in space fuzzy. All of the strangeness' for the quantum theories in different spacetime dimensions ever could be equivalent are come from the noncommutative geometry description of the quantum gravity. The exact form of the AdS/CFT is concerned with noncommutative AdS space and the classical limit of its boundary surface (on which the conformal field theory lives). This suggests that the gravity-gauge theory connection should be of the form: the string theory or $\mathrm{M}$ theory on the noncommutative background of the form $\operatorname{AdS}_{d}^{q} \times M_{D-d}^{q}$ is dual to a conformal field theory living on the classical limit of spacetime boundary. For the Type IIB string theory on the noncommutative background $\operatorname{AdS}_{5}^{q} \times S_{q}^{5}$ spectra can compare with low-order correlation functions of the $3+1$ dimensional $\mathcal{N}=4 S U(N)$ super Yang-Mills theory. The dual super Yang-Mills theory lives on the classical limit of noncommutative AdS spacetime boundary. Only both in the large $N$ and commutative limit, the string theory is weakly coupled and the supergravity is a good approximation to it. Therefore, in fact as 't Hooft suspected[1], the nature is much more crazy at the Planck scale than even string theorists could have imagined. Formalisms of string theory (gravity) on noncommutative geometry have to be constructed to gain sights of unification of gravity and quantum mechanics. 


\section{Acknowledgments}

I would like to thank Prof. J. Wess for introducing the problem to me and for enlightening discussions. I am grateful to H. Steinacker for valuable discussions. The work was supported in part by the National Science Foundation of China under Grant 19625512.

\section{References}

[1] G. 't Hooft, "Dimension reduction in quantum gravity", in Salamfest 1993, P284, gr-qc/9310026.

[2] L. Susskind, "The world as a hologram", J. Math. Phys. 36 (1995) 6377, hepth/9409089.

[3] J. Maldacena, "The large $N$ limit of superconformal field theories and supergravity", Adv. Theor. Math. Phys. 2 (1998) 231, hep-th/9711200.

[4] S. Gubser, I. Klebanov and A. Polyakov, "Gauge theory correlators from noncritical string theory", Phys. Lett. B428 (1998) 105, hep-th/9802109.

[5] E. Witten, "Anti de Sitter space and holography", Adv. Theor. Math. Phys. 2 (1998) 253, hep-th/9802150.

[6] J. Wess, " $q$-defomed phase space and its lattice structure", Int. J. Mod. Phys. 12 (1997) 4997.

[7] J. Madore, "Gravity on fuzzy space-time", ESI preprint 478 (1997), gr-qc/9709002.

[8] J. Wess and B. Zumino, "Covariant differential calculus on the quantum hyperplane", Nucl. Phys. (Proc. Suppl.) 18B (1990) 302.

[9] A. Connes, "Noncommutative Geometry", Academic Press (1994). 
[10] L. D. Faddeev, N. Yu. Reshetikhin and L. A. Takhtajan, "Quantization of Lie groups and Lie algebras", Leningrad Math. J. 1 (1990) 193.

[11] J. Klauder and B. Skagerstam, "Coherent states, applications in physics and mathematical physics", World Scientific (1985).

[12] Z. Chang, "Quantum Anti-de Sitter space", MPI-PhT 99-15, hep-th/9904091.

[13] O. Ogievetsky, "Differential operators on quantum spaces for $G L_{q}(n)$ and $S O_{q}(n)$ ", Lett. Math. Phys. 24 (1992) 245.

[14] L. Susskind and E. Witten, "The holographic bound in Anti-de Sitter space", SU-ITP-98-39, IASSNS-98-44, hep-th/9805114. 